\title{
Back to Basics: Does Benjamin Graham Filters help identify Value Stocks on Nifty $\mathbf{5 0 0}$ ?
}

\author{
Effulgence \\ Vol. 18, No. 2 \\ July - December, 2020 \\ Rukmini Devi Institute of Advanced Studies \\ E-mail : effulgence@rdias.ac.in, Website : www.rdias.ac.in \\ http://effulgence.rdias.ac.in/user/default.aspx \\ https://dx.doi.org/10.33601/effulgence.rdias/v18/i2/2020/01-12
}

\author{
Prof. Saurabh Agarwal ${ }^{1} \bowtie$ \\ Dr. Megha Agarwal ${ }^{2}$
}

\begin{abstract}
Many Investment Scholars like Graham, Schloss, Greenblatt, Neff, Lynch and Buffett have provided investment wisdom that facilitates picking value stocks from a universe of available listed stocks. In this paper, we use Graham stock selection criteria to identify value stocks. The study tested the Benjamin Graham's investing strategy from 2010 to 2020 and compared the returns with benchmark index i.e. NSE 50. It was found that Graham filters were more suitable than benchmark index in eight years out of eleven years. We also calculated Treynor ratio to map the risk-reward ratio. Treynor ratio was also higher than benchmark index for six years.
\end{abstract}

Keywords: Value Investing, Graham Filters

\section{INTRODUCTION}

B enjamin Graham (1894-1976) contributed to the literature on security analysis through his books like Security Analysis (1934) and The Intelligent Investor (1949). He has carefully discussed the issues of value investing, margin of safety, statistical analysis of securities and ownership of enterprises. He used to divide investors as either "conservative" or "aggressive". His ideas lead to the development of alternative portfolios for risk averse and risk lovers. He always advised against buying at a premium.
When the equities markets are trading at a premium, it is advisable to invest in bonds. However, it is inevitable to get return and safety of principal at the same time. There is always a compromise which has to be reached. Real estate and Insurance were considered as poor investment option by Graham.

\section{REVIEW OF LITERATURE}

Oppenheimer and Schlarbaum (1981) found that positive risk-adjusted returns were available to investor who creates portfolios based on Benjamin

1. Professor and Principal, IIF College of Commerce and Management Studies, Affiliated to CCS University, Meerut

2. Assistant Professor, Rajdhani College, University of Delhi 
Graham rules. A return of 3 to $3.5 \%$ higher than that given by index can be achieved by an investor. Investment Managers would incur very little cost by following the rules given by Graham as they are relatively easy to follow. Their results contradicted the semi-strong form of efficient markets hypothesis. Oppenheimer (1984) found that Graham's advice provided significant excess returns. The author applied ten criteria given by Graham. Stocks were listed on New York and American Stock Exchange. The results were based on data from 1974 to 1981 . Portfolios based on Graham's advice gave a mean annual return of 38 per cent compared to CRSP index of NYSE-AMEX securities. However, the performance declined post 1976, the year the criteria were published. The excess returns were found even after risk adjustment and accommodating for firm size.

$\mathrm{Vu}$ (1988) showed that the net current asset value rule developed by Graham was profitable in the 1970s and early 1980s. The results are based on 107 stocks from April 1977 to December 1984 listed on New York Stock Exchange or the American Stock Exchange. The results were true for both merged and non-merged companies' part of sample. Lauterbach and $\mathrm{Vu}$ (1993) found that Ben Graham net current asset value rule provides excess returns. However, size adjusted excess return turn out to be zero. Excess return of 25 per cent could be made as per standard market model and minus 15 per cent according to Dimson and Marsh's size-control technique. The results were based on 121 NYSE stocks during the period of 1977-1984.

Bildersee, Cheh and Zutshi (1993) tested the Benjamin Graham's NCAV model on Japanese equity markets. It was found that positive normalised NCAV portfolios outperformed Japanese equity markets. The results were sensitive to holding period of investors. The results are based on stocks listed on Tokyo Stock Exchange from April 1975 to March 1988.
Klerck and Maritz (1997) found that using Graham's criteria an investor made better returns than Industrial Index. The returns of portfolios created using Graham's filter had an annual return of 9 per cent to 12 per cent higher than the industrial index. The results are based on stocks listed on Johannesburg Stock Exchange during the period 1977 to 1994. Xiao and Arnold (2008) tested the Benjamin Graham's Net Current Asset Value Strategy in London. They found that buying stocks whose net current asset value is greater than 1.5 times the current share price creates portfolios with returns higher than that given by market. The portfolios had stocks of smaller companies and high book-to-market ratios. The results are based on stocks part of London Share Price Database (LSPD) from 1980 to 2005.

Singh and Kaur (2014) tested the Graham's stock selection criteria for a period of 15 years for stocks listed on Bombay Stock Exchange. According to them, investors can screen stocks on the basis of any five rules to identifying value maximising securities.

Zakaria and Hashim (2017) found that in Saudi Arabia, Graham's stock selection criteria enable investors in generating abnormal returns. Satisfactory earnings, financial strength and Current ratio help identify stocks that can generate abnormal returns. The results are based on data from 2000 to 2011. However, the number of companies satisfying the criteria reduced from 23 (in 2000) to just 4 (in 2011).

Rani (2019) tested Graham's and Greenblatt's Formula for 2010 to 2018. The resultant portfolios were compared on the basis of return with NSE 50 and NSE 500. It was found that portfolios based on Graham's criteria outperformed benchmarks (NSE 50 and NSE 500) in five out of 8 years under study. Portfolios based on Greenblatt's criteria outperformed the benchmark (NSE 50 and NSE 500) in 6 years out of 8 years. Portfolio Risk was also less in Graham's portfolio as compared to benchmark portfolio or the Greenblatt portfolio. Greenblatt 
criterion fared better in terms of risk-reward ratio. The Sharpe ratio, Treynor ratio, Sortino ratio and Jensen's alpha were all higher in Greenblatt portfolio as compared to either the benchmark portfolio (NSE 50 and NSE 500) or Graham's portfolio. Since most of the studies have been done internationally, there remains a need for studying the relevance of Graham's filters in emerging markets like India.

\section{RESEARCH OBJECTIVES}

a. To compare the returns of portfolio created by Graham's filter and NSE Nifty 50

b. To compare the performance of the portfolios on the basis of Treynor ratio

\section{RESEARCH METHODOLOGY}

Yearly data for NSE 500 stocks and Nifty 50 index has been extracted from CMIE prowess for a period of eleven years starting from 1st April 2010 to 31st March 2020. The yearly total returns, Price to earnings ratio, Price to Book Value Ratio, Dividend Yield, Beta, Total Assets and Paid up Equity Capital of NSE 500 stocks has been extracted. Earnings Yield has been calculated to be inverse of the Price to Earnings Ratio; Equity to Total Assets ratio has also been calculated.
Portfolios comprising of top 30 stocks for eleven different years have been created by sorting data on the basis of the following five Graham filters:

1. Price to Earnings Ratio

2. Earnings Yield

3. Equity/Total Assets Ratio

4. Price to Book Ratio

5. Dividend Yield

To sort the stocks to be included in the portfolio, the price to earnings ratio and price to book ratio are arranged in ascending order, while the earnings yield, Equity to Total Assets ratio and dividend yield are arranged in descending order. The stocks where the dividend yield was zero have been excluded from the portfolio. Graham advocates portfolios creating these filters would be able to beat the market if the rules to exit i.e. either when 50 percent returns are realized or three years whichever is earlier are met.

Table 1 depicts the stocks included in portfolios created from NSE 500 stocks in each of the eleven years from 2010-2020 by applying Graham Filters. There are certain stocks which are repeatedly meeting the Graham criteria and forming a part of the portfolio year after year.

Table 1: Portfolios Created as per Graham's Filters

\begin{tabular}{|c|l|l|l|}
\hline S.No. & \multicolumn{1}{|c|}{2010} & \multicolumn{1}{c|}{2011} & \multicolumn{1}{c|}{2012} \\
\hline 1 & Aarti Industries Ltd. & Aarti Industries Ltd. & Aarti Industries Ltd. \\
\hline 2 & Atul Ltd. & Ajanta Pharma Ltd. & Avanti Feeds Ltd. \\
\hline 3 & $\begin{array}{l}\text { Balkrishna Industries } \\
\text { Ltd. }\end{array}$ & Atul Ltd. & Bliss G V S Pharma Ltd. \\
\hline 4 & Bank Of Maharashtra & Bliss G V S Pharma Ltd. & C E S C Ltd. \\
\hline 5 & Birla Corporation Ltd. & Can Fin Homes Ltd. & Can Fin Homes Ltd. \\
\hline 6 & Can Fin Homes Ltd. & Canara Bank & Canara Bank \\
\hline 7 & Canara Bank & Capri Global Capital Ltd. & Finolex Cables Ltd. \\
\hline 8 & Central Bank Of India & Central Bank Of India & G H C L Ltd. \\
\hline 9 & Finolex Industries Ltd. & Ctd. & Garware Technical Fibres Ltd. \\
\hline 10 & G H C L Ltd. & G H C L Ltd. & Gayatri Projects Ltd. \\
\hline & & Garware Technical Fibres & Gujarat Alkalies \& Chemicals Ltd. \\
\hline
\end{tabular}




\begin{tabular}{|l|l|l|l|}
\hline 12 & Indian Bank & $\begin{array}{l}\text { Gujarat State Fertilizers \& } \\
\text { Chemicals Ltd. }\end{array}$ & $\begin{array}{l}\text { Gujarat Narmada Valley Fertilizers } \\
\text { \& Chemicals Ltd. }\end{array}$ \\
\hline 13 & Indian Oil Corpn. Ltd. & J K Paper Ltd. & $\begin{array}{l}\text { Gujarat State Fertilizers \& } \\
\text { Chemicals Ltd. }\end{array}$ \\
\hline 14 & Indian Overseas Bank & J K Tyre \& Inds. Ltd. & Indian Bank \\
\hline 15 & $\begin{array}{l}\text { J B Chemicals \& } \\
\text { Pharmaceuticals Ltd. }\end{array}$ & Jammu \& Kashmir Bank Ltd. & Indian Oil Corpn. Ltd. \\
\hline 16 & J K Cement Ltd. & K N R Constructions Ltd. & Indian Overseas Bank \\
\hline 17 & $\begin{array}{l}\text { J K Lakshmi Cement } \\
\text { Ltd. }\end{array}$ & K R B L Ltd. & J K Cement Ltd. \\
\hline 18 & J K Paper Ltd. & Magma Fincorp Ltd. & J K Lakshmi Cement Ltd. \\
\hline 19 & J K Tyre \& Inds. Ltd. & Maharashtra Seamless Ltd. & Jammu \& Kashmir Bank Ltd. \\
\hline 20 & $\begin{array}{l}\text { Jammu \& Kashmir } \\
\text { Bank Ltd. }\end{array}$ & Nava Bharat Ventures Ltd. & Kei Industries Ltd. \\
\hline 21 & K R B L Ltd. & Navin Fluorine Intl. Ltd. & Manappuram Finance Ltd. \\
\hline 22 & M R F Ltd. & Phillips Carbon Black Ltd. & Navin Fluorine Intl. Ltd. \\
\hline 23 & $\begin{array}{l}\text { Nava Bharat Ventures } \\
\text { Ltd. }\end{array}$ & $\begin{array}{l}\text { Ratnamani Metals \& Tubes } \\
\text { Ltd. }\end{array}$ & Nilkamal Ltd. \\
\hline 24 & $\begin{array}{l}\text { Navin Fluorine Intl. } \\
\text { Ltd. }\end{array}$ & S R F Ltd. & Phillips Carbon Black Ltd. \\
\hline 25 & Nilkamal Ltd. & Sonata Software Ltd. & Punjab National Bank \\
\hline 26 & Ramco Cements Ltd. & Uco Bank & Ratnamani Metals \& Tubes Ltd. \\
\hline 27 & $\begin{array}{l}\text { Ratnamani Metals \& } \\
\text { Tubes Ltd. }\end{array}$ & Uflex Ltd. & Vardhman Textiles Ltd. \\
\hline 28 & S R F Ltd. & Vinati Organics Ltd. & Suprajit Engineering Ltd. \\
\hline 29 & Uco Bank & Welspun Corp Ltd. & Uflex Ltd. \\
\hline 30 & $\begin{array}{l}\text { Vardhman Textiles } \\
\text { Ltd. }\end{array}$ & \\
\hline & & & \\
\hline
\end{tabular}

\begin{tabular}{|r|l|l|l|}
\hline S.No. & \multicolumn{1}{|c|}{2013} & \multicolumn{1}{c|}{2014} & \multicolumn{1}{c|}{2015} \\
\hline 1 & Aarti Industries Ltd. & Balmer Lawrie \& Co. Ltd. & Canara Bank \\
\hline 2 & Avanti Feeds Ltd. & Bank Of India & Capri Global Capital Ltd. \\
\hline 3 & Bank Of Maharashtra & Can Fin Homes Ltd. & Ceat Ltd. \\
\hline 4 & Bliss G V S Pharma Ltd. & Canara Bank & D C M Shriram Ltd. \\
\hline 5 & Ceat Ltd. & Capri Global Capital Ltd. & G H C L Ltd. \\
\hline 6 & D C M Shriram Ltd. & Ceat Ltd. & $\begin{array}{l}\text { Garware Technical Fibres } \\
\text { Ltd. }\end{array}$ \\
\hline 7 & $\begin{array}{l}\text { Dewan Housing Finance Corpn. } \\
\text { Ltd. }\end{array}$ & D C M Shriram Ltd. & $\begin{array}{l}\text { Gujarat Alkalies \& } \\
\text { Chemicals Ltd. }\end{array}$ \\
\hline 8 & Finolex Cables Ltd. & $\begin{array}{l}\text { Dewan Housing Finance } \\
\text { Corpn. Ltd. }\end{array}$ & $\begin{array}{l}\text { Gujarat Mineral Devp. } \\
\text { Corpn. Ltd. }\end{array}$ \\
\hline 9 & G H C L Ltd. & G H C L Ltd. & $\begin{array}{l}\text { Gujarat State Fertilizers \& } \\
\text { Chemicals Ltd. }\end{array}$ \\
\hline 10 & Garware Technical Fibres Ltd. & $\begin{array}{l}\text { Garware Technical Fibres } \\
\text { Ltd. }\end{array}$ & Himatsingka Seide Ltd. \\
\hline 11 & Gayatri Projects Ltd. & Gayatri Projects Ltd. & $\begin{array}{l}\text { Hindustan Petroleum } \\
\text { Corpn. Ltd. }\end{array}$ \\
\hline
\end{tabular}




\begin{tabular}{|c|l|l|l|}
\hline 12 & $\begin{array}{l}\text { Gujarat Alkalies \& Chemicals } \\
\text { Ltd. }\end{array}$ & $\begin{array}{l}\text { Gujarat Narmada Valley } \\
\text { Fertilizers \& Chemicals Ltd. }\end{array}$ & Hindustan Zinc Ltd. \\
\hline 13 & $\begin{array}{l}\text { Gujarat Narmada Valley } \\
\text { Fertilizers \& Chemicals Ltd. }\end{array}$ & $\begin{array}{l}\text { Gujarat State Fertilizers \& } \\
\text { Chemicals Ltd. }\end{array}$ & Indiabulls Ventures Ltd. \\
\hline 14 & $\begin{array}{l}\text { Gujarat State Fertilizers \& } \\
\text { Chemicals Ltd. }\end{array}$ & $\begin{array}{l}\text { Hindustan Petroleum } \\
\text { Corpn. Ltd. }\end{array}$ & Indian Bank \\
\hline 15 & Indian Bank & Indiabulls Ventures Ltd. & $\begin{array}{l}\text { Jammu \& Kashmir Bank } \\
\text { Ltd. }\end{array}$ \\
\hline 16 & J K Tyre \& Inds. Ltd. & Indian Bank & Jindal Saw Ltd. \\
\hline 17 & K N R Constructions Ltd. & J K Tyre \& Inds. Ltd. & Karnataka Bank Ltd. \\
\hline 18 & K P R Mill Ltd. & K N R Constructions Ltd. & N L C India Ltd. \\
\hline 19 & K R B L Ltd. & K P R Mill Ltd. & N M D C Ltd. \\
\hline 20 & Kei Industries Ltd. & K R B L Ltd. & $\begin{array}{l}\text { National Aluminium Co. } \\
\text { Ltd. }\end{array}$ \\
\hline 21 & Muthoot Finance Ltd. & P C Jeweller Ltd. & Power Finance Corpn. Ltd. \\
\hline 22 & Navin Fluorine Intl. Ltd. & Power Finance Corpn. Ltd. & R E C Ltd. \\
\hline 23 & Power Finance Corpn. Ltd. & R E C Ltd. & $\begin{array}{l}\text { Rashtriya Chemicals \& } \\
\text { Fertilizers Ltd. }\end{array}$ \\
\hline 24 & Punjab National Bank & South Indian Bank Ltd. & Reliance Infrastructure Ltd. \\
\hline 25 & R E C Ltd. & Strides Pharma Science Ltd. & S J V N Ltd. \\
\hline 26 & Ratnamani Metals \& Tubes Ltd. & Tata Steel Ltd. & Tata Steel Ltd. \\
\hline 27 & Reliance Infrastructure Ltd. & Uco Bank & Uco Bank \\
\hline 28 & S R F Ltd. & Uflex Ltd. & Uflex Ltd. \\
\hline 29 & Tata Steel Ltd. & Union Bank Of India & Union Bank Of India \\
\hline 30 & Vardhman Textiles Ltd. & Vardhman Textiles Ltd. & Welspun India Ltd. \\
\hline & & & \\
\hline
\end{tabular}

\begin{tabular}{|c|l|l|l|}
\hline S.No. & \multicolumn{1}{|c|}{2016} & \multicolumn{1}{c|}{2017} & \multicolumn{1}{c|}{2018} \\
\hline 1 & Apollo Tyres Ltd. & $\begin{array}{l}\text { Balrampur Chini Mills } \\
\text { Ltd. }\end{array}$ & Balrampur Chini Mills Ltd. \\
\hline 2 & C E S C Ltd. & $\begin{array}{l}\text { Bharat Petroleum Corpn. } \\
\text { Ltd. }\end{array}$ & $\begin{array}{l}\text { Bharat Petroleum Corpn. } \\
\text { Ltd. }\end{array}$ \\
\hline 3 & D C M Shriram Ltd. & $\begin{array}{l}\text { Chambal Fertilisers \& } \\
\text { Chemicals Ltd. }\end{array}$ & $\begin{array}{l}\text { Chennai Petroleum Corpn. } \\
\text { Ltd. }\end{array}$ \\
\hline & $\begin{array}{l}\text { Dewan Housing Finance Corpn. } \\
\text { Ltd. }\end{array}$ & $\begin{array}{l}\text { Chennai Petroleum Corpn. } \\
\text { Ltd. }\end{array}$ & D C M Shriram Ltd. \\
\hline 5 & G H C L Ltd. & Coal India Ltd. & G H C L Ltd. \\
\hline 6 & Great Eastern Shipping Co. Ltd. & D C M Shriram Ltd. & $\begin{array}{l}\text { Gujarat Alkalies \& } \\
\text { Chemicals Ltd. }\end{array}$ \\
\hline 7 & $\begin{array}{l}\text { Gujarat Alkalies \& Chemicals } \\
\text { Ltd. }\end{array}$ & $\begin{array}{l}\text { G H C L Ltd. } \\
\text { Cujarat Mineral Devp. }\end{array}$ \\
\hline 8 & $\begin{array}{l}\text { Gujarat Mineral Devp. Corpn. } \\
\text { Ctd. }\end{array}$ & $\begin{array}{l}\text { Glenmark Pharmaceuticals } \\
\text { Ltd. }\end{array}$ & $\begin{array}{l}\text { Gujarat Narmada Valley } \\
\text { Fertilizers \& Chemicals Ltd. }\end{array}$ \\
\hline 9 & $\begin{array}{l}\text { Gujarat State Fertilizers \& } \\
\text { Chemicals Ltd. }\end{array}$ & $\begin{array}{l}\text { Great Eastern Shipping } \\
\text { Co. Ltd. }\end{array}$ & H E G Ltd. \\
\hline 10 & $\begin{array}{l}\text { Hindustan Petroleum Corpn. } \\
\text { Ltd. }\end{array}$ & $\begin{array}{l}\text { Gujarat Alkalies \& } \\
\text { Chemicals Ltd. }\end{array}$ & $\begin{array}{l}\text { Hindustan Petroleum } \\
\text { Corpn. Ltd. }\end{array}$ \\
\hline 11 & Hindustan Zinc Ltd. & Heritage Foods Ltd. & $\begin{array}{l}\text { Housing \& Urban Devp. } \\
\text { Corpn. Ltd. }\end{array}$ \\
\hline
\end{tabular}




\begin{tabular}{|l|l|l|l|}
\hline 12 & J K Tyre \& Inds. Ltd. & $\begin{array}{l}\text { Hindustan Petroleum } \\
\text { Corpn. Ltd. }\end{array}$ & Indian Oil Corpn. Ltd. \\
\hline 13 & J S W Energy Ltd. & Indian Bank & J K Paper Ltd. \\
\hline 14 & Jammu \& Kashmir Bank Ltd. & Indian Oil Corpn. Ltd. & Jindal Saw Ltd. \\
\hline 15 & Jindal Saw Ltd. & J K Paper Ltd. & L I C Housing Finance Ltd. \\
\hline 16 & Karnataka Bank Ltd. & J S W Steel Ltd. & M O I L Ltd. \\
\hline 17 & Lakshmi Vilas Bank Ltd. & Jindal Saw Ltd. & $\begin{array}{l}\text { Mangalore Refinery \& } \\
\text { Petrochemicals Ltd. }\end{array}$ \\
\hline 18 & M R F Ltd. & Karnataka Bank Ltd. & Muthoot Finance Ltd. \\
\hline 19 & Muthoot Finance Ltd. & Karur Vysya Bank Ltd. & N H P C Ltd. \\
\hline 20 & National Fertilizers Ltd. & Larsen \& Toubro Infotech & N L C India Ltd. \\
\hline 21 & Oil India Ltd. & Manappuram Finance Ltd. & N M D C Ltd. \\
\hline 22 & P T C India Ltd. & N L C India Ltd. & Oil \& Natural Gas Corpn. \\
\hline 23 & Power Finance Corpn. Ltd. & Oil India Ltd. & Oil India Ltd. \\
\hline 24 & R E C Ltd. & P T C India Ltd. & P C Jeweller Ltd. \\
\hline 25 & Reliance Capital Ltd. & R E C Ltd. & P T C India Ltd. \\
\hline 26 & S J V N Ltd. & S J V N Ltd. & Power Finance Corpn. Ltd. \\
\hline 27 & South Indian Bank Ltd. & Tata Steel Ltd. & $\begin{array}{l}\text { Power Grid Corpn. Of India } \\
\text { Ltd. }\end{array}$ \\
\hline 28 & Uflex Ltd. & & R E C Ltd. \\
\hline 29 & Vardhman Textiles Ltd. & Trident Ltd. & S J V N Ltd. \\
\hline 30 & Vedanta Ltd. & Welspun India Ltd. & Vardhman Textiles Ltd. \\
\hline
\end{tabular}

\begin{tabular}{|c|l|l|}
\hline S.No. & \multicolumn{1}{|c|}{2019} & \multicolumn{1}{c|}{2020} \\
\hline 1 & Balrampur Chini Mills Ltd. & Balrampur Chini Mills Ltd. \\
\hline 2 & Bombay Dyeing \& Mfg. Co. Ltd. & Bombay Dyeing \& Mfg. Co. Ltd. \\
\hline 3 & D C M Shriram Ltd. & Chambal Fertilisers \& Chemicals Ltd. \\
\hline 4 & G H C L Ltd. & D B Corp Ltd. \\
\hline 5 & Graphite India Ltd. & G H C L Ltd. \\
\hline 6 & Gujarat Alkalies \& Chemicals Ltd. & Gayatri Projects Ltd. \\
\hline 7 & Gujarat Mineral Devp. Corpn. Ltd. & Gujarat Mineral Devp. Corpn. Ltd. \\
\hline & Gujarat State Fertilizers \& Chemicals & \\
\hline 9 & Ltd. & H E G Ltd. \\
\hline 10 & H E G Ltd. & Himatsingka Seide Ltd. \\
\hline 11 & Hindustan Petroleum Corpn. Ltd. & Housing \& Urban Devp. Corpn. Ltd. \\
\hline 12 & Indiabulls Housing Finance Ltd. & J K Paper Ltd. \\
\hline 13 & J K Paper Ltd. & Jindal Saw Ltd. \\
\hline 14 & Jindal Saw Ltd. & Karnataka Bank Ltd. \\
\hline 15 & Karnataka Bank Ltd. & Maharashtra Seamless Ltd. \\
\hline 16 & M O I L Ltd. & N C C Ltd. \\
\hline 17 & Maharashtra Seamless Ltd. & N L C India Ltd. \\
\hline & &
\end{tabular}




\begin{tabular}{|l|l|l|}
\hline 18 & N L C India Ltd. & N M D C Ltd. \\
\hline 19 & N M D C Ltd. & Nava Bharat Ventures Ltd. \\
\hline 20 & National Aluminium Co. Ltd. & Oil \& Natural Gas Corpn. Ltd. \\
\hline 21 & National Fertilizers Ltd. & P N B Housing Finance Ltd. \\
\hline 22 & Oil \& Natural Gas Corpn. Ltd. & P T C India Ltd. \\
\hline 23 & Oil India Ltd. & Phillips Carbon Black Ltd. \\
\hline 24 & P T C India Ltd. & Power Finance Corpn. Ltd. \\
\hline 25 & Phillips Carbon Black Ltd. & R E C Ltd. \\
\hline 26 & R E C Ltd. & Repco Home Finance Ltd. \\
\hline 27 & Reliance Infrastructure Ltd. & S J V N Ltd. \\
\hline 28 & S J V N Ltd. & Shriram City Union Finance Ltd. \\
\hline 29 & Tata Steel Ltd. & South Indian Bank Ltd. \\
\hline 30 & Trident Ltd. & Tata Steel Ltd. \\
\hline
\end{tabular}

Source: Author Compilation based on data from CMIE Prowess

Some of stocks which are repeatedly forming a part of our portfolio are Aarti Industries Ltd., Can Fin Homes Ltd., Canara Bank, GHCL Ltd., Gujarat State Fertilizers \& Chemicals Ltd., J K Tyre \& Inds. Ltd., Jindal Saw Ltd., NMDC Ltd., Uco Bank, Tata Steel etc.
The total returns and beta of the stocks in a portfolio are used as inputs to compute the Portfolio Return and Portfolio Beta. The Portfolio Returns and Portfolio Betas of the portfolios created as per Graham's filters are depicted in Table 2 and Table 3 respectively. The portfolios are evaluated for performance based on Treynor ratio.

Table 2 : Portfolio Returns

\begin{tabular}{|r|r|r|r|r|r|r|r|r|r|r|r|}
\hline Years & $\mathbf{2 0 1 0}$ & $\mathbf{2 0 1 1}$ & $\mathbf{2 0 1 2}$ & $\mathbf{2 0 1 3}$ & $\mathbf{2 0 1 4}$ & $\mathbf{2 0 1 5}$ & $\mathbf{2 0 1 6}$ & $\mathbf{2 0 1 7}$ & $\mathbf{2 0 1 8}$ & $\mathbf{2 0 1 9}$ & $\mathbf{2 0 2 0}$ \\
\hline 2010 & 0.94 & -1.64 & 1.77 & -0.10 & -0.70 & -0.50 & -0.36 & 1.01 & 0.56 & -1.65 & 0.15 \\
\hline 2011 & & -1.39 & 0.84 & -0.30 & -1.48 & 0.08 & -0.26 & 1.52 & 0.44 & -1.59 & 0.50 \\
\hline 2012 & & & 1.65 & -0.24 & -1.02 & 0.26 & -0.12 & 1.88 & 0.84 & -1.65 & 0.60 \\
\hline 2013 & & & & -0.02 & -1.70 & -0.18 & -0.23 & 1.30 & 1.02 & -2.01 & 0.77 \\
\hline 2014 & & & & & -2.06 & 0.39 & -0.50 & 2.02 & 0.26 & -2.29 & 1.04 \\
\hline 2015 & & & & & & 0.48 & -0.70 & 1.92 & 0.97 & -1.66 & 0.75 \\
\hline 2016 & & & & & & & -0.30 & 1.50 & 0.84 & -1.22 & 2.09 \\
\hline 2017 & & & & & & & & 0.71 & 0.52 & 0.07 & 2.58 \\
\hline 2018 & & & & & & & & & 0.25 & -0.37 & 1.69 \\
\hline 2019 & & & & & & & & & & -1.57 & 1.84 \\
\hline 2020 & & & & & & & & & & & 2.16 \\
\hline
\end{tabular}

Source: Computed by Authors based on data from CMIE Prowess 
Table 3 : Portfolio Beta

\begin{tabular}{|r|r|r|r|r|r|r|r|r|r|r|r|}
\hline Portfolios & $\mathbf{2 0 1 0}$ & $\mathbf{2 0 1 1}$ & $\mathbf{2 0 1 2}$ & $\mathbf{2 0 1 3}$ & $\mathbf{2 0 1 4}$ & $\mathbf{2 0 1 5}$ & $\mathbf{2 0 1 6}$ & $\mathbf{2 0 1 7}$ & $\mathbf{2 0 1 8}$ & $\mathbf{2 0 1 9}$ & $\mathbf{2 0 2 0}$ \\
\hline 2010 & 1.01 & 1.03 & 1.02 & 1.02 & 1.17 & 1.22 & 1.19 & 1.22 & 1.25 & 1.26 & 1.19 \\
\hline 2011 & & 1.05 & 1.04 & 1.05 & 1.16 & 1.19 & 1.22 & 1.22 & 1.28 & 1.38 & 1.33 \\
\hline 2012 & & & 1.04 & 1.06 & 1.18 & 1.27 & 1.29 & 1.35 & 1.38 & 1.42 & 1.33 \\
\hline 2013 & & & & 1.16 & 1.23 & 1.27 & 1.30 & 1.32 & 1.35 & 1.43 & 1.35 \\
\hline 2014 & & & & & 1.21 & 1.42 & 1.44 & 1.48 & 1.51 & 1.59 & 1.46 \\
\hline 2015 & & & & & & 1.37 & 1.41 & 1.47 & 1.52 & 1.56 & 1.44 \\
\hline 2016 & & & & & & & 1.32 & 1.37 & 1.40 & 1.41 & 1.29 \\
\hline 2017 & & & & & & & & 1.22 & 1.26 & 1.30 & 1.24 \\
\hline 2018 & & & & & & & & & 1.24 & 1.26 & 1.22 \\
\hline 2019 & & & & & & & & & & 1.42 & 1.42 \\
\hline 2020 & & & & & & & & & & & 1.42 \\
\hline
\end{tabular}

Source: Computed by Authors based on data from CMIE Prowess

\section{EMPIRICAL RESULTS AND ANALYSIS}

As per Graham's filters the portfolio created in 2010 generates returns (0.94) higher than the index returns for the same year (0.72). Graham advocates exiting if a return more than $50 \%$ is realized. Hence, we must exit the portfolio in the first year itself as the goal has been realized. The 2011 portfolio, 2013 portfolio, 2014 portfolio give a negative return in the first year vis a vis an index return of 8.9 percent. It is not able to beat the market and hence we would exit in the maximum allowed period of three years. The 2012 portfolio generates a high return in the first year (1.65) compared to the negative index returns $(-0.08)$.

The Graham portfolio created in year 2015 generate returns (1.7) higher than index returns (0.12) if held for three years as suggested by Graham. The same applies to 2016 portfolio, 2017 portfolio, 2018 portfolio and 2020 portfolio. The 2019 portfolio is also able to generate a return higher than the index in its two years holding period.

The Graham portfolios are able to generate a higher return than the index either in the first year or three years of their holding time. This however was not true for three of the eleven portfolios created for the years 2011, 2013 and 2014.

Table 3 depicts the Graham's portfolios systematic risk as measured by beta. Portfolio beta has been computed as it is more relevant than the total risk (variance/standard deviation) for diversified portfolios like here. The portfolio betas are consistently higher than 1 depicting a higher risk than the index. However, this increased risk is compensated with a higher return generated by these portfolios. Table 4 presents the closing prices, returns and beta for the Nifty 50 which is used as a proxy of the market for our study. This data has been extracted from CMIE prowess for a period of 1st April 2009- 31st March 2020. Returns are calculated by using the formula (Rt - Rt-1)/ Rt-1. The mean market (index) returns for Nifty 50 are a little higher than 13 percent (13.09) for the eleven years period under consideration. The systematic risk is lesser too (0.93) in comparison to the portfolios created using Graham's filters. 
Table 4 : Index Returns and Beta

\begin{tabular}{|c|c|c|c|}
\hline \multicolumn{5}{|c|}{ Closing Price } & Return & Beta \\
\hline Nifty 50 & 5278 & 0.724837 & 0.98 \\
\hline 2010 & 5749.5 & 0.089333 & 0.97 \\
\hline 2011 & 5248.15 & -0.0872 & 0.94 \\
\hline 2012 & 5930.2 & 0.12996 & 0.92 \\
\hline 2013 & 6696.4 & 0.129203 & 0.88 \\
\hline 2014 & 8181.5 & 0.221776 & 0.92 \\
\hline 2015 & 7849.8 & -0.04054 & 0.92 \\
\hline 2016 & 9304.05 & 0.185259 & 0.92 \\
\hline 2017 & 10739.35 & 0.154266 & 0.92 \\
\hline 2018 & 11748.15 & 0.093935 & 0.92 \\
\hline 2019 & 9859.9 & -0.16073 & 0.92 \\
\hline 2020 & Mean Returns & 0.130918 & 0.93 \\
\hline 11 years & & & \\
\hline
\end{tabular}

Source : Computed by Authors based on data from CMIE Prowess

The portfolios created using Graham filters were able to provide higher returns than the market if the rules for exit are followed (return higher than 0.5 or three years holding period whichever is earlier) for eight of the eleven portfolios. This shows that Graham filters are able to beat the Indian stock markets when stocks are chosen from the NSE 500 stocks.

To further substantiate our results we conducted Mann-Whitney $U$ test to see if the returns of our portfolio are significantly different from the returns generated by the Nifty 50 index. To compute the returns of the portfolio again the Graham exit rules are followed. When more than 50 percent returns are realized in the first year itself, these returns become the portfolio returns. When more than 50 percent returns are realized in the first two years we average returns of these two years to find portfolio returns. Else, the returns for three years of holding period are averaged to compute the portfolio returns as this is the maximum time an investor can hold a Graham portfolio. Table 5 depicts the portfolio returns as well as index returns used for comparison. It also shows the portfolio beta computed following the same exit rules.

Table 5 : Returns and Systematic Risk

\begin{tabular}{|c|c|c|c|c|}
\hline Years & Portfolios Returns & Index Returns & Portfolio Beta & Index Beta \\
\hline 2010 & 0.94267 & 0.72484 & 1.01 & 0.98 \\
\hline 2011 & -0.28344 & 0.08933 & 1.05 & 0.97 \\
\hline 2012 & 1.65467 & -0.08720 & 1.04 & 0.94 \\
\hline 2013 & -0.63356 & 0.12996 & 1.22 & 0.92 \\
\hline 2014 & -0.72078 & 0.12920 & 1.36 & 0.88 \\
\hline 2015 & 0.56478 & 0.22178 & 1.42 & 0.92 \\
\hline 2016 & 0.60150 & -0.04054 & 1.35 & 0.92 \\
\hline 2017 & 0.70733 & 0.18526 & 1.22 & 0.92 \\
\hline 2018 & 0.52544 & 0.15427 & 1.24 & 0.92 \\
\hline 2019 & 0.13250 & 0.09393 & 1.42 & 0.92 \\
\hline 2020 & 2.16433 & -0.16073 & 1.42 & 0.92 \\
\hline
\end{tabular}

Source: Computed by Authors based on data from CMIE Prowess 
As our sample size was lesser than 30 and to relax the normality conditions in the data, we performed Mann-Whitney $U$ test to compare the returns generated by Graham's portfolios and market index Nifty 50. This was done using SPSS 20. Equal numbers of observations (11 each) were used in the analysis. Table 6 presents the results of the statistical tests conducted. It clearly shows the mean rank of returns generated by Graham's portfolio (13.27) to be considerably higher than the mean rank of the returns generated by the market index Nifty 50 (9.73). This means Graham's portfolios were able to generate a higher return than in the index return for a period of 2010-2020. However, these results are not statistically significant at a significance level of 5 percent. Thus we don't have any evidence to conclude that the returns generated using Graham's filters are better than the returns generated by the market index Nifty 50 using the 2 sample independent Mann-Whitney U test.

Table 6 : Results of Mann-Whitney U Test on Returns

\begin{tabular}{|l|l|r|r|r|}
\hline \multirow{5}{*}{ Returns } & Groups & N & Mean Rank & Sum of Ranks \\
\cline { 2 - 5 } & Graham & & & \\
\cline { 2 - 4 } & Portfolio & 11 & 13.27 & 146.00 \\
\cline { 2 - 5 } & Market & 11 & 9.73 & 107.00 \\
\cline { 2 - 5 } & Index & & & \\
\cline { 2 - 5 } & Total & 22 & & \\
\hline
\end{tabular}

\begin{tabular}{|l|r|}
\hline \multicolumn{2}{|c|}{ Test Statistics } \\
\hline Mann-Whitney U & \multicolumn{1}{c|}{ Returns } \\
\hline Wilcoxon W & 41.000 \\
\hline Z & 107.000 \\
\hline Asymp. Sig. (2-tailed) & -1.280 \\
\hline Exact Sig. [2*(1-tailed Sig.)] & .200 \\
\hline
\end{tabular}

Source: Computed by Authors based on data from CMIE Prowess and SPSS 20

To further compare the performance of our portfolios with the market portfolio, we calculate the 'Trenyor ratio'. This ratio is a reward to risk ratio with excess return generated by the portfolio over risk free rate in the numerator and systematic risk (beta) as the denominator.

Treynor Ratio $=\left[\right.$ Portfolio Return $\left(\mathrm{R}_{\mathrm{p}}\right)-$ Risk free Return $\left.\left(\mathrm{R}_{\mathrm{f}}\right)\right]$ / Portfolio Beta $\left(\beta_{\mathrm{P}}\right)$

The return on 364 days Treasury bill is taken as a proxy for the risk free return. This is 6.5 percent as on 1st April 2019. We have calculated the ratios again based on Graham's exit rules. When at least 50 percent return is generated in the first year itself we take the return and beta of only the first year for ratio calculation as the investor would exit here. If at least 50 percent returns are generated in the first two years then returns of these two years are averaged for ratio calculation. Else, the returns of three years are averaged as this is the maximum period for which the investor would hold a Graham portfolio. The corresponding Treynor ratios for Nifty 50 are also computed so as to facilitate a comparison between Graham's portfolios and the market. The Treynor ratios are presented in Table 7. 
Table 7 : Treynor Ratios

\begin{tabular}{|c|c|c|}
\hline Years & Portfolios Trenyor Ratio & Markets Trenyor Ratio \\
\hline 2010 & 0.86498 & 0.67330 \\
\hline 2011 & -0.03699 & 0.02509 \\
\hline 2012 & 1.53542 & -0.16191 \\
\hline 2013 & -0.06348 & 0.07061 \\
\hline 2014 & -0.19280 & 0.07296 \\
\hline 2015 & 0.03923 & 0.17041 \\
\hline 2016 & 0.09956 & -0.11472 \\
\hline 2017 & 0.52780 & 0.13072 \\
\hline 2018 & 0.04128 & 0.09703 \\
\hline 2019 & 0.04754 & 0.03145 \\
\hline 2020 & 1.47529 & -0.24536 \\
\hline
\end{tabular}

Source: Author Calculations

We can see that the Graham portfolio does better than the market for the years 2010, 2012, 2016, 2017, 2019 and 2020. In fact in the year 2020 when the market performance as per Treynor ratio is negative, the Graham portfolio is able to generate high positive returns. In these years the Graham portfolios were able to outperform the market. Our results are sensitive to investors holding period. However, following Graham's strategy not all investors were profitable as the Treynor ratios were negative occasionally for the investment period 2011, 2013 and 2014.

\section{CONCLUSIONS}

Like Oppenheimer and Schlarbaum (1981), Vu (1988), Lauterbach and Vu (1993), Bildersee, Cheh and Zutshi (1993), Klerck and Maritz (1997), Xiao and Arnold (2008), Singh and Kaur (2014), Zakaria and Hashim (2017) and Rani (2019), we found that portfolios based on Graham's filters outperformed the index. However, our results are sensitive to investment holding period. This shows that Graham filters can be used by active players like Financial Institutions, Mutual Funds and Investment Advisors. Holding Graham's portfolios passively would not yield positive returns over long run. Out of the eleven year, the returns for three years were negative exhibiting that Graham's filters can facilitate optimal portfolio creation but in some years the returns may turn out to be negative. The portfolio beta was also higher for portfolio's created using Graham's filters. This clearly shows higher risk in portfolios based on Graham's filters. When we evaluate performance of portfolios based on Treynor ratio, we observe that portfolio based on Graham's filters perform better in six out of eleven years.

\section{REFERENCES}

Bildersee, J. S., Cheh, J. J., \& Zutshi, A. (1993). The performance of Japanese common stocks in relation to their net current asset values. Japan and the World Economy, 5(3), 197-215.

Klerck, W. G., \& Maritz, A. C. (1997). A test of Graham's stock selection criteria on industrial shares traded on the JSE. Investment Analysts Journal, 26(45), 25-33.

Lauterbach, B. and J. D. Vu. (1993). Ben Graham's Net Current Asset Value Rule Revisited: The Size-Adjusted Returns. Quarterly Journal of Business and Economics, 32(1), 82-108.

Mahesh, Prashant, 2020 (in press), In flight to safety, Bharat Bond ETF trumps other fixed-income products, ET Bureau, Retrieved from

https:/ / economictimes.indiatimes.com/markets/bo nds/in-flight-to-safety-bharat-bond-etf-trumpsother-fixed-income-products / articleshow / 
75057609.cms?utm_source $=$ contentofinterest\&ut m_medium $=$ text\&utm_campaign $=$ cppst

Oppenheimer, H. R. and G. G. Schlarbaum. (1981). Investing with Ben Graham: An Ex Ante Test of the Efficient Markets Hypothesis. The Journal of Financial and Quantitative Analysis, 16(3), 341360.

Oppenheimer, H. R. (1984). A Test of Ben Graham's Stock Selection Criteria. Financial Analysts Journal, 40(5), 68-74.

Rani,P. (2019). Risk-Reward Agility of the Benjamin Graham and Joel Greenblatt's Investing Philosophy in the Indian Stock Market. International Journal of Multidisciplinary Research, 4(3), 492 - 498.

Singh, J., \& Kaur, K. (2014). Testing Ben Graham's Stock Selection Criteria in Indian Stock Market. Management and Labour Studies, 39(1), 43-62.

Vu, J. D. (1988), An Empirical Analysis of Ben Graham's Net Current Asset Value Rule. The Financial Review, 23(2), 215-225.

Xiao, Y., \& Arnold, G. C. (2008). Testing Benjamin Graham's Net Current Asset Value Strategy in London. The Journal of Investing, 17(4), 11-19.

Zakaria, N. and F. Hashim. (2017). Emerging Markets: Evaluating Graham's Stock Selection Criteria on Portfolio Return in Saudi Arabia Stock Market. International Journal of Economics and Financial Issues, 7(2), 453-459. 